\title{
Prevalence of bifid median nerves and persistent median arteries and their association with carpal tunnel syndrome in a sample of latino poultry processors and other manual workers
}

\author{
FRANCIS O. WALKER, MD ${ }^{1,2}$, MICHAEL S. CARTWRIGHT, MS, MD ${ }^{1,2}$, JILL N. BLOCKER, \\ MS $^{3}$, THOMAS A. ARCURY, PhD ${ }^{2,4}$, JUNG IM SUK, MD ${ }^{5}$, HAIYING CHEN, PhD ${ }^{2,3}$, MARK R. \\ SCHULTZ, PhD ${ }^{6}$, JOSEPH G. GRZYWACZ, PhD ${ }^{2,4}$, DANA C. MORA, MPH ${ }^{7}$, and SARA A. \\ QUANDT, PhD 2,7 \\ ${ }^{1}$ Department of Neurology, Wake Forest School of Medicine, Medical Center Boulevard, Winston- \\ Salem, North Carolina 27157-1078, USA \\ ${ }^{2}$ Center for Worker Health, Wake Forest School of Medicine, Winston-Salem, North Carolina, \\ USA \\ ${ }^{3}$ Department of Biostatistical Sciences, Division of Public Health Sciences, Wake Forest School \\ of Medicine, Winston-Salem, North Carolina, USA \\ ${ }^{4}$ Department of Family and Community Medicine, Wake Forest School of Medicine, Winston- \\ Salem, North Carolina, USA \\ ${ }^{5}$ Department of Neurology School of Medicine, Catholic University of Daegu, Daegu, South Korea \\ ${ }^{6}$ Department of Public Health Education, University of North Carolina at Greensboro, Greensboro, \\ North Carolina, USA \\ ${ }^{7}$ Department of Epidemiology and Prevention, Division of Public Health Sciences, Wake Forest \\ School of Medicine, Winston-Salem, North Carolina, USA
}

\section{Abstract}

Introduction-The prevalence of bifid median nerves and persistent median arteries, their cooccurrence, and their relationship to carpal tunnel syndrome (CTS) are only understood partially.

Methods-We screened 1026 wrists of 513 Latino manual laborers in North Carolina for bifid median nerves and persistent median arteries using electrodiagnosis and ultrasound.

\footnotetext{
Results-A total of $8.6 \%$ of wrists had a bifid median nerve, and $3.7 \%$ of wrists had a persistent median artery independent of subgroup ethnicity, age, gender, or type of work. An association with definite carpal tunnel syndrome was not found. The presence of either anatomic variant was associated with a high likelihood of co-occurrence of another variant in the same or the contralateral wrist.
}

(C) 2013 Wiley Periodicals, Inc.

Correspondence to: F.O. Walker; fwalker@wakehealth.edu. 
Conclusions-The occurrence of median anatomic variants can be determined in field studies using ultrasound. Persistent median arteries and bifid median nerves tend to co-occur but do not put manual laborers at additional risk of developing CTS.

\section{Keywords}

entrapment neuropathy; epidemiology; neuromuscular ultrasound; occupational health; poultry workers

Knowledge of a bifid median nerve and a persistent median artery may be of importance in the diagnosis and treatment of entrapment neuropathies at the wrist. Bifid median nerves may be independent risk factors for the development of carpal tunnel syndrome (CTS), because they tend to have a relatively higher cross-sectional area than non-bifid median nerves and occupy more room in the carpal tunnel. ${ }^{1}$ Persistent median arteries also occupy space in the carpal tunnel and are known to cause CTS when they dilate or become thrombosed. ${ }^{2-4}$

The prevalence of bifid median nerves and persistent median arteries in the general population is delineated poorly. The reported prevalence of bifid median nerves has ranged from $2 \%$ to $26 \%$ per wrist. ${ }^{1,5-8}$ This lack of consistency may result from variation in measurement technique (e.g., magnetic resonance imaging or ultrasound) or the patient population studied (Table 1). Far more prevalence studies, many in cadavers, have focused on persistent median arteries, yet prevalence estimates remain wide-ranging, varying from $0.6 \%$ to $30 \%$ based on primary and secondary reports. ${ }^{9-14}$ In part, this may be explained by the effect of sample population or age, in that neonatal studies have identified a high prevalence of persistent median artery. ${ }^{11-13}$ Recent studies have used ultrasound and magnetic resonance imaging (MRI) and have reported results within the broad range defined in cadaveric studies. 5,6

In this study of a large population of Latino manual workers, we investigated the prevalence of bifid median nerve and persistent median arteries, the likelihood of the coexistence of these 2 anatomic variants, and their relationship to CTS through screening with highresolution ultrasound, hand diagrams, and electrodiagnostic testing.

\section{METHODS}

This study was done as part of a larger study of the occupational health in Latino manual laborers, age $\geq 18$ years, who work in poultry processing and non-poultry processing occupations at least 35 hours per week in 4 western North Carolina counties. Recruitment has been described in more detail elsewhere. ${ }^{15}$ Briefly, community-based sampling was done in areas with a high proportion of Latino residents in order to recruit self-identified adult Latino manual laborers who were divided into those working in the poultry processing industry versus other forms of manual work. Recruited participants underwent a 1-hour-long interview and subsequently attended a data collection clinic. The clinics occurred on 7 Sundays in 2009 and 2010. All participants provided informed consent, and the protocol was approved by the institutional review board of the Wake Forest School of Medicine. Participants were paid $\$ 40$. Of the 1526 subjects screened, 957 were eligible for 
participation. Of these, 742 underwent interviews, and 518 attended the data collection clinics; 5 subjects left before undergoing nerve conduction studies. Thus, 1036 wrists were screened with ultrasound in 518 participants, and 1026 wrists (513 participants) were available to analyze the relationship of bifid nerves or persistent median arteries to electrodiagnostically defined CTS.

\section{Ultrasound Evaluations}

Ultrasound evaluations were conducted with a MyLab 25 Gold Instrument (Esaote North America, Inc., Indianapolis, Indiana) using a 12-18-MHZ linear-array transducer. Assessment was performed by examiners skilled in ultrasound imaging of the median nerve and its variants (F.W. and M.C.) and blinded to the results of other evaluations in the study. Two examiners were available at all times to review images for which questions arose. Participants were seated and placed their hands, fully supinated, on the examination table for evaluation. The nerve was examined in multiple views with the wrist and fingers in flexion, extension, and neutral positions, and images of each median nerve were stored in digital files. All bifid nerves and those with persistent median arteries were identified using realtime and color Doppler imaging.

\section{Nerve Conduction Studies and Diagnosis of CTS}

Nerve conduction studies (NCS), performed by experienced technicians blinded to other information, have been described in more detail elsewhere. ${ }^{15}$ After warming the hands to $32^{\circ} \mathrm{C}$, antidromic median and ulnar sensory studies were conducted with ring electrodes on digits 2 and 5 with wrist stimulation $140 \mathrm{~mm}$ proximal. Onset and peak latencies were recorded. Median motor studies were conducted in participants when sensory responses were absent.

The diagnoses of possible or definite CTS were based on a combination of symptoms (Katz hand diagram) and electrodiagnostic studies. ${ }^{15}$ The Katz hand diagrams were scored according to standardized methods as 0 (unlikely), 1 (possible), 2 (probable), or likely (3), by 2 clinicians (F.W. and M.C.) blinded to NCS and ultrasound results. For this study, subjects were assigned a symptom score of 1 when the hand diagram rating was 1,2 , or 3 . All others were assigned a symptom score of 0 . Subjects with a median-ulnar peak latency difference of $>0.79 \mathrm{~ms}$ were given an NCS score of 2; if it was $>0.49 \mathrm{~ms}$, they were scored 1. All others were scored 0 . If the NCS and symptom score added to 0 , then the subject was defined as having no CTS. If the scores added to 1 or 2 , they were rated as possible CTS; those adding to 3 were graded as definite CTS. We developed 2 separate definitions of CTS for this study. The first was a strict definition of CTS, which consisted only of subjects scoring a total of 3 , and a less rigorous definition of those subjects scoring 1,2, or 3 .

\section{Statistical Analysis}

The goal of the analysis was to assess the prevalence of bifid median nerves and persistent median arteries in the study population to evaluate what personal characteristics predict the presence/absence of each anatomical trait, and to test whether these traits predict CTS. Descriptive statistics were calculated as mean and standard deviation of continuous variables and percentage and frequency of discrete outcomes. Using information from ultrasound, a 
binary variable was created to reflect the presence/absence of a bifid median nerve in either (yes in right or left or both) or neither (no) wrist. The same measure was created for a persistent median artery. Bivariate associations between these 2 outcomes and the discrete participant characteristics of job status, gender, and spoken language were calculated using a chi-square test of association, whereas the continuous characteristics of age, height, weight, and body mass index (BMI) were assessed using a Student $t$-test. Simple and multivariate logistic regression modeling accounting for site strata were used to assess the relationship between the presence of a persistent median artery or bifid median nerve and CTS. Mixed effects models were also adjusted for the clustering effect of dwelling, participant, and hand to account for within-participant correlation. Multivariate models included gender and age as fixed effects with adjusted odds ratios and $P$-values being reported. Level of significance was set at $P<0.05$, and all analyses were performed using SAS, version 9.2 (SAS Institute, Cary, North Carolina).

\section{RESULTS}

We found a bifid median nerve in $8.6 \%$ of wrists and a persistent median artery in $3.7 \%$. Ten patients had bilateral bifid median nerves, so the prevalence per individual was $13.3 \%$. Four patients had bilateral persistent median arteries, so the prevalence per individual was $5.8 \%$. No significant differences in the frequency of a median artery or bifid median nerve were noted between manual laborers in poultry and non-poultry occupations. Likewise, no significant differences were noted with age, gender, weight, height, or subgroup ethnicity (as evidenced by self-identified native language) (Table 2).

The presence of either a bifid median nerve or persistent median artery in 1 wrist increased the likelihood of encountering similar variants in the same or other wrist (Fig. 1). The effect was pronounced, particularly with median arteries. A bifid left median nerve almost tripled the probability of having a bifid right median nerve or a persistent left median artery; it quintupled the probability of having a persistent right median artery. Significantly more persistent left median arteries ( $n=22)$ were found than right $(n=16 ; P=0.0032)$.

Two common patterns of bifid nerve and median arteries were seen in this population. In 1, the nerve was split by the median artery, and in the other the median artery was located on the periphery of the nerve (Fig. 2). The bifid nerve can be divided by a large fibrous tissue barrier, or a small septum.

We examined the prevalence of carpal tunnel syndrome in participants with either an ipsilateral persistent median artery or a bifid median nerve compared to those without these anatomical findings. With a strict definition of CTS, there was no association. Even with a less rigorous definition of CTS, the difference was not significant $(P=0.0547)$. A multivariate analysis accounting for body weight, gender, and age was also not significant $(P=0.0695)$ (Table 3$)$.

\section{DISCUSSION}

In this study of $>1000$ wrists we found prevalence rates of bifid median nerve and persistent median arteries within the ranges previously reported (Table 1$).{ }^{1-14}$ The ranges reported 
were wide and varied significantly with technique (MRI, ultrasound, dissection) and population studied (neonatal and adult cadavers, healthy subjects by ultrasound, or patients imaged for non-CTS disorders). Some evidence suggests that the prevalence of persistent median arteries is higher in neonates, but other factors that influence the prevalence of either persistent median artery or bifid median nerve have not been identified. For example, it is unknown whether the presence of these abnormalities is higher or lower in manual laborers than in the general population. Our study showed no consistent effect of adult age or gender on the occurrence of persistent median arteries, but a slightly lower occurrence in the right hand. This finding is of uncertain significance and may reflect the small number of affected hands overall; it has not been found in other studies of persistent median arteries. It is possible that those engaged in heavy manual labor may be more prone to thrombosis and involution of a persistent median artery, an effect that may be exaggerated in the dominant hand.

The population we studied, adult Latino manual workers, may be of some relevance in that certain anatomical variants in the forearm vary widely in different ethnic populations. An absent palmaris tendon, for example, occurs in $65 \%$ of Turkish, $25 \%$ of European, and $4.4 \%$ of East African populations. ${ }^{16-19}$ Further studies are needed to ascertain whether ethnic differences influence the wide range of reported prevalence of persistent median arteries and bifid median nerves. Of interest, almost all the Latinos in this study were born in villages in Mexico and Central America, which tend to have ethnic differences denoted by native language. In primarily Spanish-speaking villages the population is predominantly Mestizo, of mixed Native American and European (e.g., Spanish) ancestry. In villages in which an indigenous language is spoken, the population is predominantly Native American. We found no significant differences in the occurrence of neurovascular anatomical variants of the forearm in these two groups.

Our findings suggest there is a robust relationship between the presence of a persistent median artery and a contralateral median artery and either ipsilateral or contralateral bifid median nerves. There is a somewhat less robust relationship between a bifid median nerve and a contra-lateral bifid nerve and either ipsilateral or contralateral persistent median arteries. The association of a right bifid median nerve with a left persistent median artery appears somewhat equivocal. However, given the overall findings, and the multiple comparisons, it would be difficult to draw firm conclusions about this relationship. Bifid nerves were found to be about twice as frequent as persistent median arteries, and the cooccurrence of these traits suggests that they share a common embryological or genetic origin. It may be that later resorption of median arteries during embryogenesis or maturation, particularly those that split the median nerve, tend to leave a residual small fibrous partition or gap. If so, this could help explain why the median arteries and bifid nerves are interrelated.

It is difficult to compare prevalence findings regarding median arteries and bifid median nerves from prior studies using MRI angiography or cadaveric dissection with ultrasound studies. For example, it is possible that ultrasound is more sensitive for detection of persistent median arteries that are patent than those that are non-patent; for example, a nonpatent or thrombosed median artery can have the appearance of a small nerve fascicle. ${ }^{4}$ It is 
not known if 1 of these techniques is more sensitive than another for detecting these anatomical variants. Furthermore, MRI studies have suggested that bifid nerves may be categorized into those apparent in the palm versus those apparent in the carpal tunnel. ${ }^{7}$ Because our studies concentrated on the proximal one third of the carpal tunnel, studies looking at more distal portions of the nerve may encounter a somewhat higher prevalence of this anomaly. Absolute percentages of persistent median arteries or bifid median nerves may vary with the techniques used to detect them.

The relationship of bifid median nerves and persistent median arteries to CTS is also of interest. Our study found no evidence to suggest that an ipsilateral median artery or bifid nerve increases the risk of CTS. Previous studies and many single case reports have suggested a stronger relationship between CTS and these wrist anomalies. ${ }^{1-14}$ It should be noted, however, that those studies looked at patients referred to a physician for evaluation of suspected carpal tunnel syndrome, in contrast to the general worker population that was screened in our study. The presence of a persistent median artery or bifid median nerve does not substantially increase the risk of CTS in manual laborers. An earlier analysis in this population, however, showed that type of work had a significant impact on the prevalence of CTS. Of the poultry workers studied, $8.7 \%$ had definite CTS compared with only $4.0 \%$ in other types of manual work (odds ratio $2.51,95 \%$ confidence interval $1.8-3.5, P<0.0001$ ). ${ }^{15}$

This study has the limitations common to other large epidemiological studies of CTS in terms of extrapolating findings to patients seen in the context of typical office or diagnostic laboratory setting. Unlike patients, these study participants were an unselected sample of Latino manual workers who were not specifically seeking medical attention. Furthermore, the clinical and electrodiagnostic evaluations were less complete than those typically performed in a traditional medical setting. Nonetheless, there is considerable epidemiological precedent that establishes the reliability and validity of the approach chosen for screening for the prevalence of CTS in this population. The use of ultrasound to detect bifid median nerves and persistent median arteries may have some limitations with respect to other modalities. For example, cadaveric dissection under magnification may be more sensitive than ultrasound imaging in detecting anatomical anomalies. However, cadaveric studies are not representative of a healthy, working population and neither are retrospective radiological studies. Prospective, high-resolution MRI studies may be desirable, but the cost would be prohibitive.

Strengths of this study include its large number of participants; the defined population investigated; and the consistency, availability, cost-effectiveness, and validity of the measures chosen. Our findings provide robust evidence that age, gender, and type of manual labor performed are unrelated to the presence or persistence of median arteries or bifid median nerves, and we have highlighted the feasibility of using ultrasound in the field to identify the prevalence of anatomic variants at the wrist and their associations with workrelated disorders. The discovery of either a bifid median nerve or a persistent median artery significantly increases the likelihood of identifying a persistent median artery in either hand or a bifid median nerve in the contralateral hand. Whereas the type of work performed significantly influences the likelihood of developing CTS in a large population of Latino manual laborers, the presence of persistent median arteries or bifid median nerves does not. 


\section{Acknowledgments}

This study was supported by the National Institute for Occupational Safety and Health (R01 OH9251).

\section{Abbreviations}

$\begin{array}{ll}\text { BMI } & \text { body mass index } \\ \text { BMN } & \text { bifid median nerve } \\ \text { CTS } & \text { carpal tunnel syndrome } \\ \text { NCS } & \text { nerve conduction studies }\end{array}$

\section{References}

1. Bayrak IK, Bayrak AO, Kale M, Turker H, Diren B. Bifid median nerve in patients with carpal tunnel syndrome. J Ultrasound Med. 2008; 27:1129-1136. [PubMed: 18645070]

2. Khashaba A. Carpal tunnel syndrome from thrombosed persistent median artery. J Emerg Med. 2002; 22:55-57. [PubMed: 11809556]

3. Feldkamp MM, Gentili F, Hudson AR, Guha A. A persistent median artery causing carpal tunnel syndrome in a patient with chronic renal failure: case report. Neurosurgery. $1995 ; 37: 140-143$ [PubMed: 8587676]

4. Walker FO, Li Z, Lyles M. Sheet fitting palsy. J Clin Neuromuscul Dis. 2012; 14:48-50. [PubMed: 22922583]

5. Pierre-Jerome C, Smitson RD Jr, Shah RK, Moncayo V, Abdelnoor M, Terk MR. MRI of the median nerve and median artery in the carpal tunnel: prevalence of their anatomical variations and clinical significance. Surg Radiol Anat. 2010; 32:315-322. [PubMed: 20033168]

6. Granata G, Caliandro P, Pazzaglia C, Minciotti I, Russo G, Martinoli C, et al. Prevalence of bifid median nerve at wrist assessed through ultrasound. Neurol Sci. 2011; 32:615-618. [PubMed: 21533564]

7. Lannicelli E, Chianta GA, Salvini V, Almberger M, Monacelli G, Passariello R. Evaluation of bifid median nerve with sonography and MR imaging. J Ultrasound Med. 2000; 19:481-485. [PubMed: 10898302]

8. Padua L, Liotta G, Di Pasquale A, Granata G, Pazzaglia C, Caliandro P, et al. Contribution of ultrasound in the assessment of nerve diseases. Eur J Neurol. 2012; 19:47-54. [PubMed: 21554493]

9. Coleman SS, Anson BJ. Arterial patterns in the hand based upon a study of 650 specimens. Surg Gynecol Obstet. 1961; 113:409-424. [PubMed: 13694610]

10. Rodríguez-Niedenfuhr M, Sanudo JR, Vãzquez T, Nearn L, Logan B, Parkin I. Median artery revisited. J Anat. 1999; 195:57-63. [PubMed: 10473293]

11. Kopuz C, Baris S, Gulman B. A further morphological study of the persistent median artery in neonatal cadavers. Surg Radiol Anat. 1997; 19:403-406. [PubMed: 9479715]

12. Olave E, Prates JD, Gabrielli C, Pardi P. Median artery and superficial palmar branch of the radial artery in the carpal tunnel. Scand J Plast Reconstr Surg Hand Surg. 1997; 31:13-16. [PubMed: 9075283]

13. Ahn DS, Yoon ES, Koo SH, Park SH. A prospective study of the anatomic variations of the median nerve in the carpal tunnel in Asians. Ann Plast Surg. 2000; 44:282-287. [PubMed: 10735220]

14. Gassner EM, Schocke M, Peer S, Schwabegger A, Jaschke W, Bodner G. Peristent median artery in the carpal tunnel: color Doppler ultrasonographic findings. J Ultrasound Med. 2002; 21:455461. [PubMed: 11934102]

15. Cartwright MS, Walker FO, Blocker JN, Schulz MR, Arcury TA, Grzywacz JG, et al. The prevalence of carpal tunnel syndrome in Latino poultry-processing workers and other Latino manual workers. J Occup Environ Med. 2012; 54:198-201. [PubMed: 22258161] 
16. Ceyhan O, Mavt A. Distribution of agenesis of plamaris longus muscle in 12-18 year old age groups. Indian J Med Sci. 1997; 51:156-160. [PubMed: 9355719]

17. Thompson NW, Mockford BJ, Cran GW. Absence of the palmaris longus muscle: a population study. Ulster Med J. 2001; 70:22-24. [PubMed: 11428320]

18. Ndou R, Gangata H, Mitchell B, Ngcongo T, Louw G. The frequency of absence of plamaris longus in a South African population of mixed race. Clin Anat. 2010; 23:437-442. [PubMed: 20235176]

19. Kigera JW, Mukwaya S. Frequency of agenesis of palmaris longus through clinical examinationan East African study. PloS One. 2011; 6(12):e28997. [PubMed: 22174943] 


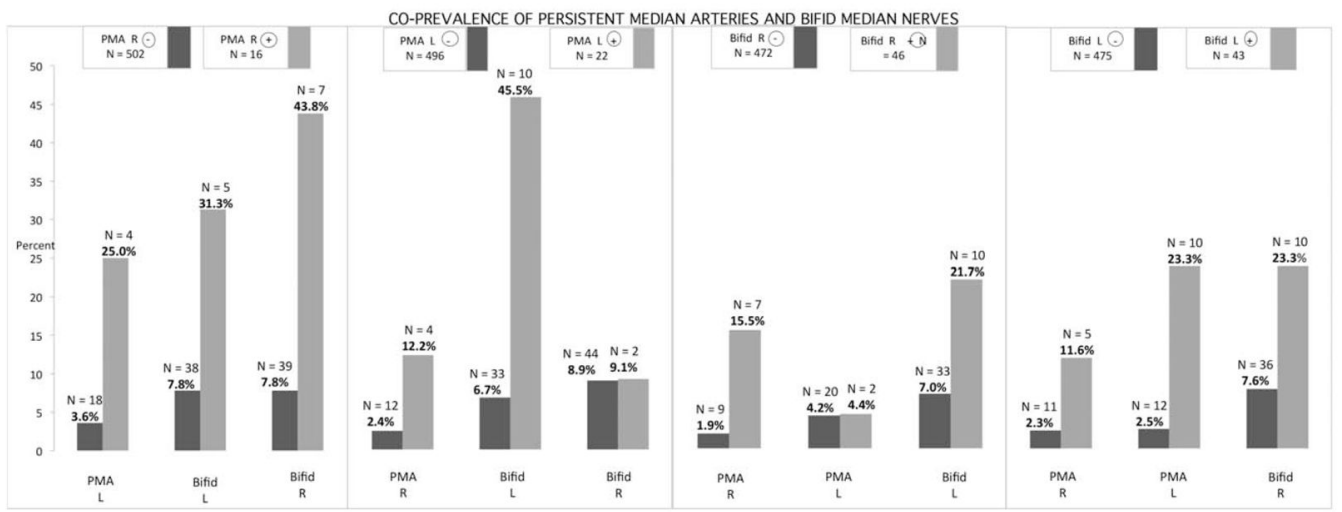

FIGURE 1.

Degree to which bifid median nerves and persistent median arteries cosegregate. At the top of the figure are the numbers of study participants with each specific abnormality. Below, the bar graph illustrates the frequency of co-occurrence of this abnormality with the other abnormalities listed. For example, there are 16 participants who have a right persistent median artery (PMA). Of these, 7 also have a bifid right median nerve (7 of 16, or $44 \%$ ). There are 502 participants without a right persistent median artery, and in this group 39 subjects have a right bifid median nerve (39 of 502, or 7.8\%). PMA, persistent median artery; bifid, bifid median nerve. 


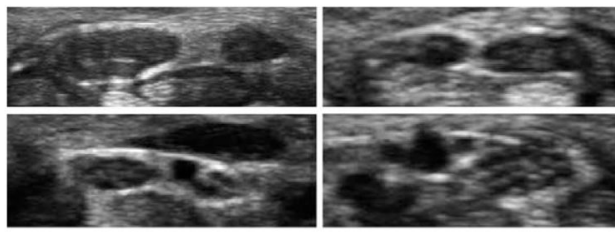

A

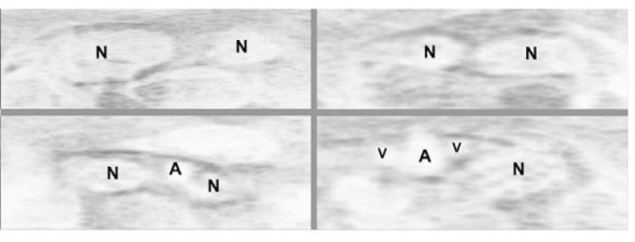

B

FIGURE 2.

(A) Composite image of 4 separate median nerves from the wrists of 4 different participants in the study, cropped and aligned to display the most common variations of bifid median nerves and persistent median arteries. (B) Negative image of these structures, labeled to show the different types of splitting seen with bifid median nerves. In the upper left image, the 2 branches of the nerve $(\mathrm{N})$ are divided by a thick fibrous tissue barrier; in the upper right image, this barrier is much narrower; in some cases the barrier is a very thin partitioning membrane. In the lower left image, the barrier contains a persistent median artery (A). In the lower right image, the artery flanks the nerve, which is not bifid, but the artery (A) is accompanied by 2 smaller veins (V). Although a flanking artery is a common finding with persistent median arteries, it is rare to see persistent median veins. 


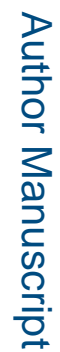

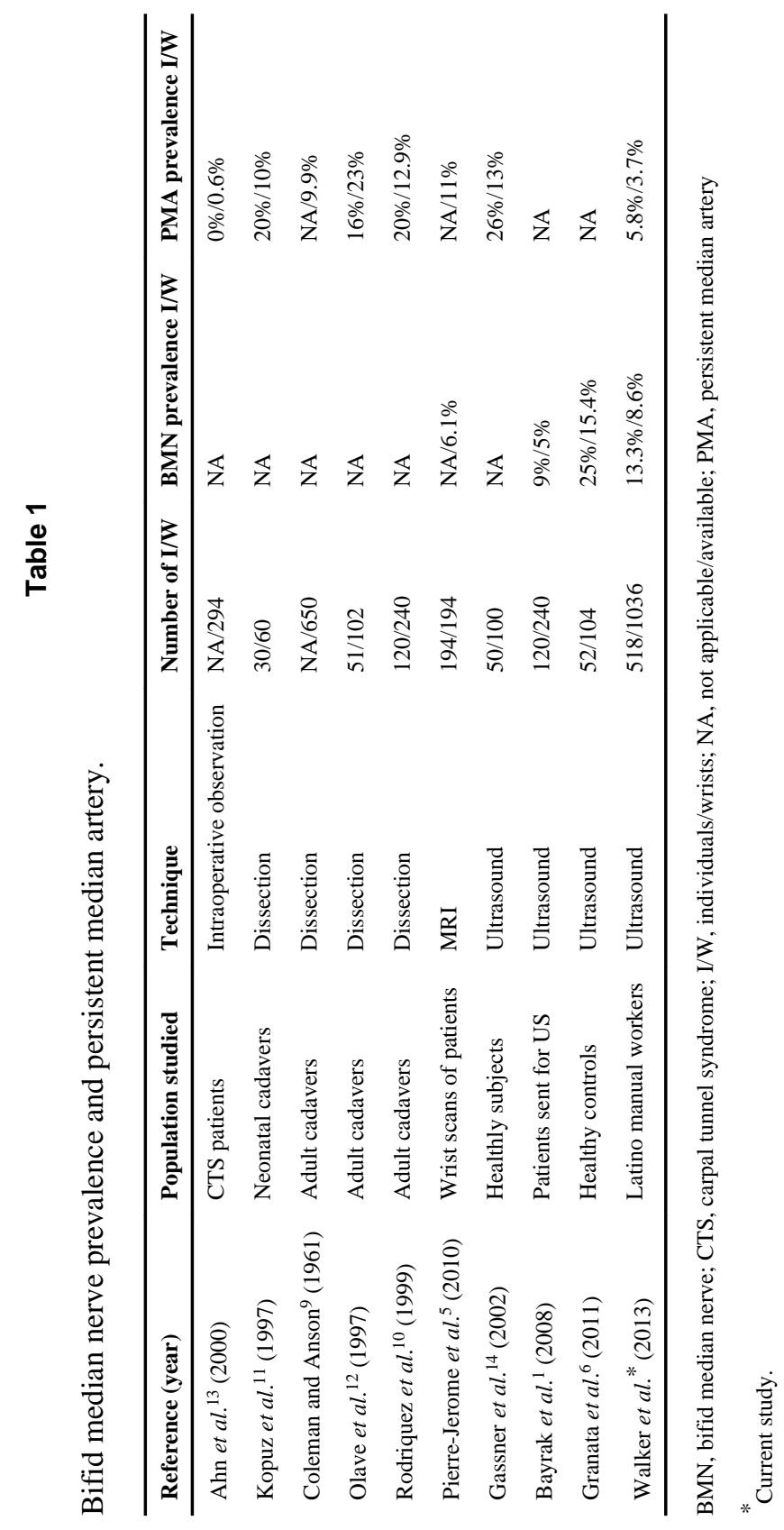

Muscle Nerve. Author manuscript; available in PMC 2013 November 21. 


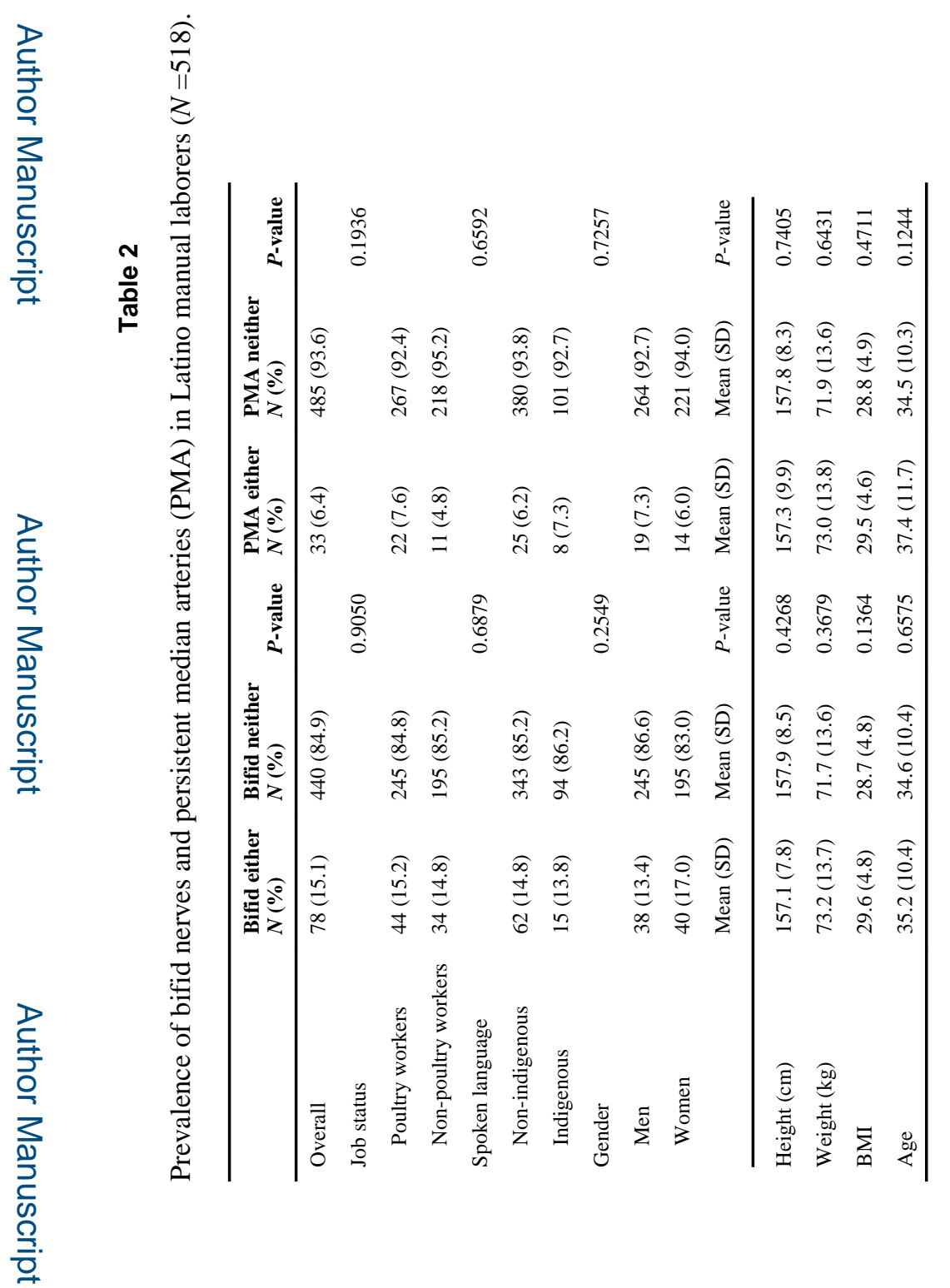

Muscle Nerve. Author manuscript; available in PMC 2013 November 21. 


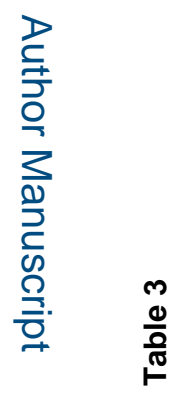

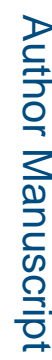

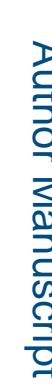

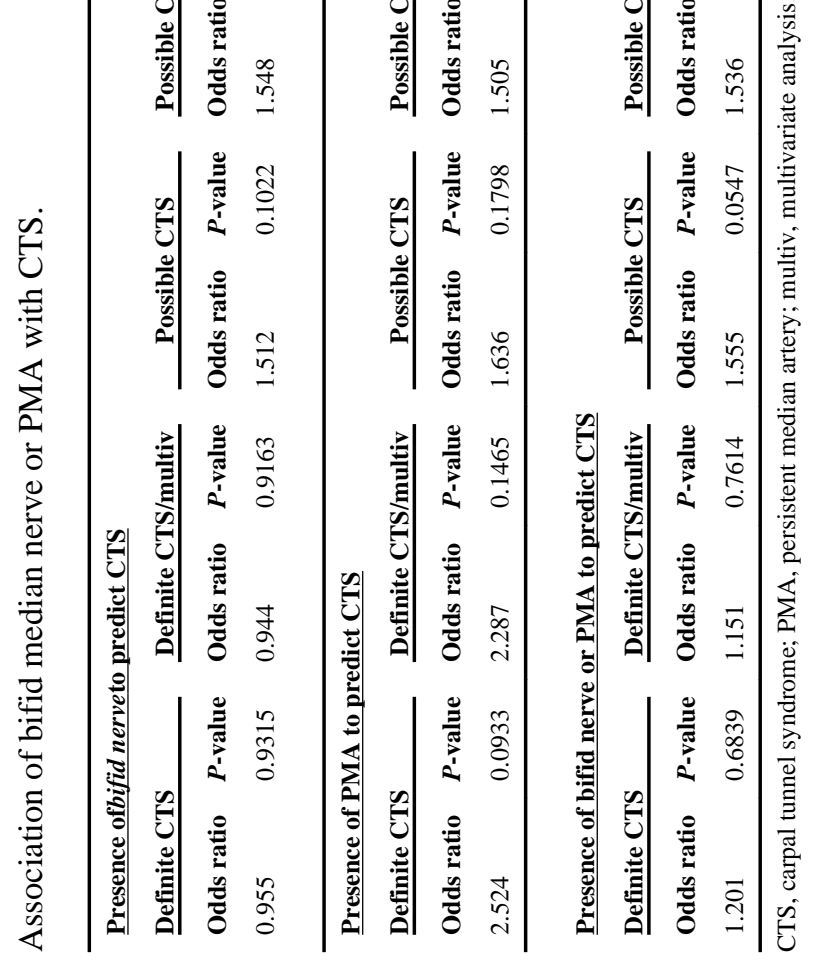

Muscle Nerve. Author manuscript; available in PMC 2013 November 21. 